\title{
Quantitative analysis of MRI-guided attenuation correction techniques in time-of-flight brain PET/MRI
}

\author{
Abolfazl Mehranian ${ }^{1}$, Hossein Arabi ${ }^{1}$ and Habib Zaidi ${ }^{1,2,3}$ \\ ${ }^{1}$ Division of Nuclear Medicine and Molecular Imaging, Geneva University Hospital, Geneva, \\ Switzerland \\ ${ }^{2}$ Geneva Neuroscience Centre, University of Geneva, Geneva, Switzerland \\ ${ }^{3}$ Department of Nuclear Medicine and Molecular Imaging, University of Groningen, Groningen, \\ Netherlands
}

\section{Corresponding author:}

Habib Zaidi, Ph.D

Division of Nuclear Medicine and Molecular Imaging, Geneva University Hospital CH-1211 Geneva, Switzerland

Tel: +41223727258

Fax: +4122372 7169

E-mail: habib.zaidi@hcuge.ch

Running title: MRI-guided attenuation correction in brain PET 


\section{ABSTRACT}

Purpose: In quantitative PET/MR imaging, attenuation correction (AC) of PET data is markedly challenged by the need of deriving accurate attenuation maps from MR images. A number of strategies have been developed for MRI-guided attenuation correction with different degrees of success. In this work, we compare the quantitative performance of three generic AC methods, including standard 3class MR segmentation-based, advanced atlas-registration-based and emission-based approaches in the context of brain time-of-flight (TOF) PET/MRI.

Materials and Methods: Fourteen patients referred for diagnostic MRI and ${ }^{18} \mathrm{~F}-\mathrm{FDG}$ PET/CT brain scans were included in this comparative study. For each study, PET images were reconstructed using four different attenuation maps derived from CT-based AC (CTAC) serving as reference, standard 3class MR-segmentation, atlas-registration and emission-based AC methods. To generate 3-class attenuation maps, T1-weighted MRI images were segmented into background air, fat and soft-tissue classes followed by assignment of constant linear attenuation coefficients of $0,0.0864$ and $0.0975 \mathrm{~cm}^{-}$

${ }^{1}$ to each class, respectively. A robust atlas-registration based AC method was developed for pseudoCT generation using local weighted fusion of atlases based on their morphological similarity to target MR images. Our recently proposed MRI-guided maximum likelihood reconstruction of activity and attenuation (MLAA) algorithm was employed to estimate the attenuation map from TOF emission data. The performance of the different AC algorithms in terms of prediction of bones and quantification of PET tracer uptake was objectively evaluated with respect to reference CTAC maps and CTAC-PET images.

Results: Qualitative evaluation showed that the MLAA-AC method could sparsely estimate bones and accurately differentiate them from air cavities. It was found that the atlas-AC method can accurately predict bones with variable errors in defining air cavities. Quantitative assessment of bone extraction accuracy based on Dice similarity coefficient (DSC) showed that MLAA-AC and atlas-AC resulted in DSC mean values of 0.79 and 0.92 , respectively, in all patients. The MLAA-AC and atlas-AC methods predicted mean linear attenuation coefficients of 0.107 and $0.134 \mathrm{~cm}^{-1}$, respectively, for the skull compared to reference CTAC mean value of $0.138 \mathrm{~cm}^{-1}$. The evaluation of the relative change in tracer uptake within 32 distinct regions of the brain with respect to CTAC PET images showed that the 3-class MRAC, MLAA-AC and atlas-AC methods resulted in quantification errors of $-16.2 \pm 3.6 \%$, $13.3 \pm 3.3 \%$ and $1.0 \pm 3.4 \%$, respectively. Linear regression and Bland-Altman concordance plots showed that both 3-class MRAC and MLAA-AC methods result in a significant systematic bias in PET tracer uptake, while the atlas-AC method results in a negligible bias.

Conclusion: The standard 3-class MRAC method significantly underestimated cerebral PET tracer uptake. While current state-of-the-art MLAA-AC methods look promising, they were unable to noticeably reduce quantification errors in the context of brain imaging. Conversely, the proposed atlasAC method provided the most accurate attenuation maps, and thus the lowest quantification bias.

Keywords: PET/MRI, brain imaging, quantification, attenuation correction, segmentation. 


\section{INTRODUCTION}

Positron emission tomography (PET) and magnetic resonance imaging (MRI) provide complementary information about the physiology, function and morphology of different organs/tissues in normal and pathological states. This hybrid imaging technology proved to be valuable in a number of clinical indications, particularly in the assessment of brain disorders and in neuroscience research. Recent developments in integrated hybrid PET/MRI systems have streamlined the simultaneous acquisition of PET and MRI data and therefore the spatiotemporal correlation of the information provided by each modality alone. The availability of numerous PET radiotracers and advanced contrast-enhanced MR imaging techniques makes simultaneous PET/MRI especially attractive for multiparametric monitoring of physiopathological variations associated with brain disorders in vivo (Garibotto et al., 2013), staging and characterization of tumours (Jacobs et al., 2005) and quantitative measurement of neural activity and brain hemodynamics (Catana et al., 2012).

Despite these advantages and still unexplored potential avenues for PET/MRI technology, the quantification of PET and MRI tracer concentrations in PET/MRI studies is currently one of the most involved and challenging tasks encountered by these modalities. For instance, the concentration of MR contrast agents in dynamic contract-enhanced MRI studies is affected by the underlying native T1 relaxation time of tissues and, as such, it is not linearly related to signal intensities. It is therefore necessary to design and perform additional MR sequences to calculate quantitative T1 maps of tissues and thereby to quantify the tracer concentration. Similarly, the quantification of PET radiotracer uptake in PET/MRI is challenged by the need of deriving underlying photon linear attenuation coefficients (LACs) of tissues from MR images. In contrast to x-ray computed tomography (CT) used on PET/CT scanners, MR image intensities are not directly related to the photon attenuating properties of tissues (i.e. atomic number, electron density) but rather to their magnetic properties (i.e. T1 and T2 relaxation times, proton density) as well as MR pulse timing parameters (i.e. flip angle, repetition time (TR), echo time (TE), inversion time (TI) ... etc. (Zaidi, 2007). Hence, the direct conversion of MRI intensities to $511 \mathrm{KeV}$ attenuation maps using experimentally derived mapping functions is not feasible.

Since the introduction of PET/MRI in clinical practice, segmentation-based AC methods were adopted to derive attenuation maps where MR images are segmented into a number of tissue classes and predefined LACs are assigned to each tissue class (Martinez-Möller et al., 2009). To achieve reliable PET quantification, MR images should ideally be segmented into 6 tissue classes, namely, soft-tissue, fat, lung, air, cortical and spongeous bones (Ouyang et al., 2013, Akbarzadeh et al., 2013a). However, a major challenge is the differentiation between bones and air cavities in the skull and surrounding soft-tissue in the vertebra, since bones do not exhibit detectable signals when using conventional MRI sequences mainly due to their short T2 relaxation time. In commercial PET/MRI scanners, bones are thus currently replaced by soft-tissues. However, neglecting bone in the resulting 
attenuation maps can give rise to substantial errors in quantification of brain PET images $5-10 \%$ (Andersen et al., 2014), 10-29\% (Dickson et al., 2014)) and lesions seated close to bones (2-31\% (Samarin et al., 2012, Arabi et al., 2015)). In brain PET/MRI imaging, ultrashort echo-time (UTE) (Keereman et al., 2010, Catana et al., 2010) and more recently zero echo-time (ZTE) (Wiesinger et al., 2015, Delso et al., 2015) MR sequences have been developed to specifically delineate bones and include them in the attenuation maps. Initial UTE-based AC studies have reported quantification errors of less than 5\% (Aasheim et al., 2015); however, the inhomogeneous and imprecise classification of bones, particularly in the presence of diamagnetic susceptibility effects at air/bone or air/soft-tissue interfaces (Delso et al., 2014) can give rise to errors in the range 4-17\% in different regions of the brain (Dickson et al., 2014). Recently, Delso et al. demonstrated that ZTE-based bone segmentation outperforms its UTE-based counterpart with reduced segmentation errors (Delso et al., 2015). However, these two MRI sequences are time consuming (between 3-6 min for low and high resolution U/ZTE acquisitions (Delso et al., 2015)), which currently limits their adoption in clinical practice. The assignment of constant LACs to each tissue class is another source of error in segmentation-based AC techniques, since inter/intra-patient variability of attenuation coefficients is not accounted for.

Alternative AC approaches have therefore been explored in the context of brain imaging including registration-based and emission-based AC methods (Zaidi et al., 2007). In registration-based methods, MRI of an atlas and co-registered CT (or transmission) are registered to the patient's MR image and then the CT or transmission maps are transformed using the same transformation fields to produce patient-specific attenuation map (Kops et al., 2009, Montandon and Zaidi, 2005). Hofmann et al. proposed to combine atlas-based registration and Gaussian regression processing based on local similarities to increase the robustness to local miss-registration and derive more accurate patientspecific brain attenuation maps (Hofmann et al., 2008). Quantitative analysis demonstrated that their proposed approach reduces the errors to less than $3 \%$ on average.

Burgos et al. proposed a pseudo-CT synthesization method using multi-atlas registration and local weighting of aligned CT-MRI atlases using a local image similarity measure, such as local normalized cross-correlation (LNCC) (Burgos et al., 2014). Comparison with UTE segmentation-based AC using 42 brain datasets showed that this method results in a mean relative error of $<1 \%$, while the UTEbased approach resulted in a mean error of $12 \%$. The authors recently improved and validated their method using two different PET radiotracers for brain imaging reporting a mean error of $<2 \%$. Recently, Izquierdo-Garcia et al. improved atlas registration through segmentation of the target MR images into 6 distinct regions (using statistical parametric mapping software) followed by its registration to a CT/MRI atlas space (Izquierdo-Garcia et al., 2014). For the atlas dataset, a CT template is calculated by averaging the co-registered CT images. A pseudo-CT is then generated by inverse transformation of the template CT images into the patient's space. Quantitative evaluation using 15 patients revealed that this method results in a mean error of $<4 \%$ in tracer uptake. 
Emission-based AC methods have also been recently revisited exploiting time-of-flight (TOF) PET/MRI and PET/CT imaging (Salomon et al., 2011). In this so-called maximum likelihood reconstruction of activity and attenuation (MLAA) approach (Nuyts et al., 1999), the attenuation and PET activity maps are simultaneously estimated from TOF emission data. These AC methods are particularly promising for deriving patient-specific attenuation maps. Salomon et al. proposed to constrain the estimation of attenuation coefficients over many anatomical regions obtained from MR images, thereby improving the robustness of the MLAA algorithm. However, theoretical and experimental studies showed that the attenuation maps can be estimated from emission data up to a scaling factor (Defrise et al., 2012, Rezaei et al., 2012). Mehranian and Zaidi improved the estimation of attenuation maps using a Gaussian mixture model (GMM) constrained by spatial MR and statistical CT information (Mehranian and Zaidi, 2015b). The quantitative analysis using clinical whole-body studies showed that the proposed MLAA-AC method resulted in $-10.2 \%$ quantification error in bony structures compared to $-18.4 \%$ induced by the 4 -class MRAC method. For a more detailed survey of the strategies devised to address the challenges of AC in PET/MRI, interested readers are referred to recent reviews on the topic (Mehranian et al., 2015, Bezrukov et al., 2013).

There is growing evidence emphasizing the need to develop advanced AC methods capable of detecting or predicting bones in MR-guided derivation of attenuation maps, particularly for neurological studies. In this work, we compare the performance of segmentation-, atlas-registrationand emission-based attenuation correction methods using clinical brain PET/MRI studies to shed the light on the limitations and potential of the latter two methods in the prediction of bones. To the best of our knowledge, this is the first clinical study in which atlas registration-based and emission-based AC methods are juxtaposed and compared with each other. UTE-based AC methods were not included, since their performance has been compared with atlas-based AC methods elsewhere (Burgos et al., 2014). We focus on the PET quantitative performance of various AC methods with respect to the reference CT-based AC (CTAC)-PET using region and voxel-wise analyses.

\section{MATERIALS AND METHODS}

\subsection{PET/CT and MRI data acquisition}

PET/CT and MRI brain datasets of 14 patients $(64.6 \pm 11.7$ yrs) from Geneva University Hospital were retrospectively employed for the quantitative evaluation of the three generic MRI-guided attenuation correction methods, namely, the 3-class-AC, atlas-AC and MLAA-AC. The clinical indication of the studies was dementia (70\%), epilepsy (25\%) and grading of brain tumours (5\%). As a first-line diagnostic step, the patients underwent an MRI scan on a 3T Siemens MAGNETOM Skyra scanner (Siemens Healthcare, Erlangen, Germany) with a 64 channel head coil. The MRI scans included a 3D T1-weighted magnetization-prepared rapid gradient-echo, MP-RAGE (TE/TR/TI, 2.3 $\mathrm{ms} / 1900 \mathrm{~ms} / 970 \mathrm{~ms}$, flip angle $8^{\circ} ; \mathrm{NEX}=1$, voxel size $0.8 \times 0.8 \times 0.8 \mathrm{~mm}^{3}$ ) and a $2 \mathrm{D} \mathrm{T} 2$-weighted 
turbo spin-echo, TSE (TE/TR, $100 \mathrm{~ms} / 6200 \mathrm{~ms}, \mathrm{NEX}=2$; voxel size $0.4 \times 0.4 \times 4 \mathrm{~mm}^{3}$ ). In the next step, the patients underwent an ${ }^{18} \mathrm{~F}$-FDG PET/CT scan on the Biograph mCT scanner (Siemens Healthcare, Erlangen, Germany). The data were acquired for 20 minutes after injection of $210.2 \pm 13.9$ $\mathrm{MBq}{ }^{18}$ F-FDG. A brain CTAC scan was performed for PET attenuation correction using the following parameters: $120 \mathrm{kVp}, 20 \mathrm{mAs}, 0.3 \mathrm{sec}$. rotation speed, voxel size $0.9 \times 0.9 \times 2.5 \mathrm{~mm}^{3}$.

\subsection{Attenuation map generation}

To generate the MRI-guided attenuation maps, T1- and T2-weighted MR images of each patient were non-rigidly registered to corresponding CTAC-PET images using the Hermes multimodality brain registration software (Hermes medical solutions AB, Sweden). The field-of-view (FOV) and voxel size of the resulting MR images were then matched with CT images using the Elastix registration tool (Klein et al., 2010). As described in the following sub-sections, the registered MR images were used for generation of segmentation-based MRAC map and to guide atlas-based and emission-based attenuation prediction.

\subsubsection{CT-based and segmentation-based 3-class MRAC maps}

For quantitative comparison of the MRI-guided AC methods against a reference AC method, CTbased attenuation maps were generated for each patient using Siemens e7 tools. CT Hounsfield units were converted to 511-keV attenuation coefficients using a $\mathrm{kVp}$-dependent bilinear mapping approach (Carney et al., 2006). The $512 \times 512$ attenuation maps are then matched with the FOV of PET and downsized to a resolution of $400 \times 400$. Finally, they were smoothed to the resolution of PET images using an isotropic Gaussian filter (4 mm full-width at half-maximum).

In neurological PET/MRI studies, brain MR images are typically segmented into 3 tissue classes: soft-tissue, fat and background, and if possible internal, air tissue classes. In this retrospective study, MR images have not been acquired for attenuation correction using the conventional Dixon fat and water sequence but rather for diagnostic purpose using high-resolution T1 MP-RAGE and T2 TSE sequence. Since adipose tissues have short T1 relaxation times, the fat tissue class existing in the scalp and neck area was segmented from T1-weighted images using a heuristically defined threshold. The outside air background was obtained from the reference CTAC images in order to minimize the errors induced by mis-registration between MRI and PET/CT datasets. The soft-tissue class was then defined as the complement of fat and outside air tissue classes. By this procedure, all bones and internal air cavities and metal-induced susceptibility artefacts were ignored and classified as soft-tissue. Mean linear attenuation coefficients of $0,0.864$ and $0.0975 \mathrm{~cm}^{-1}$ were assigned to the background, fat and soft-tissue classes, respectively. The resulting attenuation maps were downsized to the resolution of CTAC maps and superimposed by the corresponding CT bed attenuation map.

\subsubsection{Atlas-based AC}


The atlas database was constructed using pairs of T1-weighted MR and CT brain images. In the first step, MR images were denoised using anisotropic diffusion filtering following intra-patient intensity inhomogeneity correction utilizing N4 bias correction (Tustison et al., 2010). Then, inter-patient intensity non-uniformity was addressed using histogram matching. As shown in Figure 1, all MRIs in the atlas database are then registered to the target MR image. This inter-subject coordinate mapping was obtained using a combination of rigid registration based on normalized mutual information and non-rigid registration as described previously (Akbarzadeh et al., 2013b). All the CT images in the atlas database were then mapped to the target MR image using the same transformation that maps the subject's corresponding MR image to the target subject. By these registration and mapping processes, a series of MRI/CT pairs aligned to the MRI of the target subject is obtained.

Given a series of MRI/CT pairs, each aligned CT can be considered as a pseudo-CT candidate of the target subject. In order to improve the quality of resulting pseudo-CTs, the proposed atlas fusion framework consists of two consecutive steps. In the first step, the atlas fusion is locally optimized based on the accuracy of bone identification and then the atlases are locally weighted and fused based on their morphological similarity to the target MR image and accuracy of bone estimation. To do so, we use $M_{n}$ to denote aligned training MR images with the corresponding bone label maps $L_{n}$ obtained from simple thresholding of aligned CT images using a value of 140 Hounsfield units (HUs). We assume the label maps take a discrete value of 1 for bony regions and 0 elsewhere. The goal of the first step is to estimate the bone label map $(\widehat{B})$ associated with the target MR image $(T)$. This can be achieved via maximum-a-posteriori (MAP) estimation:

$$
\begin{aligned}
\hat{B} & =\arg \max _{L} p\left(L \mid T ;\left\{M_{n}, L_{n}\right\}\right) \\
& =\arg \max _{L} p\left(L, T ;\left\{M_{n}, L_{n}\right\}\right)
\end{aligned}
$$

The second term denotes the joint probability of the label map and image intensity given the training data, where $n$ is the index of training subjects in the atlas dataset. In the following, we make the assumption that the image intensity values $M_{n}(x)$ and labels $L_{n}(x)$ at each voxel $(x)$ are conditionally independent, which leads to the following formulation:

$$
\hat{B}(x)=\arg \max _{L} \sum_{n=1}^{N} p_{n}\left(T(x) ; M_{n}(x)\right) p_{n}\left(B(x) ; L_{n}(x)\right)
$$

where $N$ is the number of subjects in the atlas dataset. Here we call the first term in equation (2) $p_{n}\left(T(x) ; M_{n}(x)\right)$ image morphology likelihood and the second term $p_{n}\left(B(x) ; L_{n}(x)\right)$ label prior. The main objective in defining image morphology likelihood is to assign higher weights to similar atlases in such a way that the similarity measurement is robust to intensity variation across patients that and features must represent information about the structures in the image. Phase congruency map (PCM) has been shown to be a robust image feature (Kovesi, 2000). In PCM, the local Fourier components of the image are all in phase (congruent) in locations where there are meaningful edges in the image. A PCM can be used to detect structural characteristics of an image in a way that is invariant to image 
intensity and robust to noise (Kovesi, 2000). Equation (3) calculates the phase congruency of an image at location $x$ where $E n_{l o c}(x)$ represents the local energy of the image, $\operatorname{Tr}$ is an offset to avert the effect of noise on the calculation of the local energy, $F_{m}$ indicates the amplitude of the $m^{\text {th }}$ Fourier component, and $\varepsilon$ is a small value used to avoid division by zero (Kovesi, 2000, Ortiz and Martel, 2012).

$$
P C M(x)=\frac{\left\lfloor E n_{l o c}(x)-T r\right\rfloor}{\sum_{m} F_{m}(x)+\varepsilon}
$$

We adopt a Gaussian distribution with a stationary variance $\sigma^{2}$ as the image morphology likelihood term based on phase congruency of target and atlas MR images as:

$$
p_{n}\left(T(x) ; M_{n}(x)\right)=\frac{1}{\sqrt{2 \pi \sigma^{2}}} \exp \left[-\frac{1}{2 \sigma^{2}}\left(P C M(T(x))-P C M\left(M_{n}(x)\right)\right)^{2}\right]
$$

Moreover, we define the label prior term $p_{n}\left(B(x) ; L_{n}(x)\right)$ based on the signed distance transform from the bone label map $\left(L_{n}\right)$ in the atlas data set which is assumed to be positive inside the bony structures.

$$
p_{n}\left(B(x) ; L_{n}(x)\right)=\frac{1}{N r(x)} \exp \left(\rho D_{n}(x)\right)
$$

where $D_{n}(x)$ denotes the signed distance transform of the bone label map in training subject $n, \rho>0$ is the slope constant, and $\operatorname{Nr}(x)$ is the partition function.

By inserting the image morphology likelihood (Eq. 4) and label prior (Eq. 5) terms in Eq. (2), the optimization problem can be solved by for each voxel independently. The fused label of each voxel is computed via a local weighted voting strategy. The local image morphology likelihood terms serve as weights and the label prior values serve as votes. Therefore, at each voxel, training images that are more similar to the target image at this voxel after registration are given higher weighted. The output of this step $(\hat{B})$ is the most likely bone segmentaion of the target MR image. In the next step, this bone segmentation is used to assess the performance and define weighting factors for each of the aligned atlas images. The atlas fusion is achieved using equation (6) where $A C T_{n}(x)$ is the CT value of the $n^{\text {th }}$ atlas image at voxel $x$ (Figure 1).

$$
\operatorname{PCT}(x)=\frac{\sum_{n=1}^{N} \omega_{n}(x) \times A C T_{n}(x)}{\sum_{n=1}^{N} \omega_{n}(x)}
$$

where $\omega_{n}(x)$ is the weighting factor determining the contribution of the $n^{\text {th }}$ atlas at voxel $x$ to generate the final pseudo-CT (PCT) which is defined as:

$$
\left.\omega_{n}(x)=\frac{1}{\sqrt{2 \pi \alpha^{2}}} \exp \left[-\frac{1}{2 \alpha^{2}}\left(P C M(T(x))-P C M\left(I_{n}(x)\right)\right)^{2}\right] \times \frac{1}{\sqrt{2 \pi \delta^{2}}} \exp \left[-\frac{1}{2 \delta^{2}}\left(D_{n}(x)\right)-D_{B}(x)\right)^{2}\right]
$$

Here $D_{B}$ indicates the signed distance transform of the obtained bone label map of the target image $(\widehat{B})$. The first term in Eq. (7) measures the morphological similarity between the $n^{\text {th }}$ MRI atlas and target MR image while the second term assesses the performance of the $n^{\text {th }}$ CT atlas image with respect to the estimated bone segmentation of the target MR image $(\hat{B})$. The two Gaussian 
distributions are adjusted using stationary variances $\alpha^{2}$ and $\delta^{2}$. These free parameters in the above equation were optimized via leave-one-out cross-validation (LOOCV) and parameter sweeping.

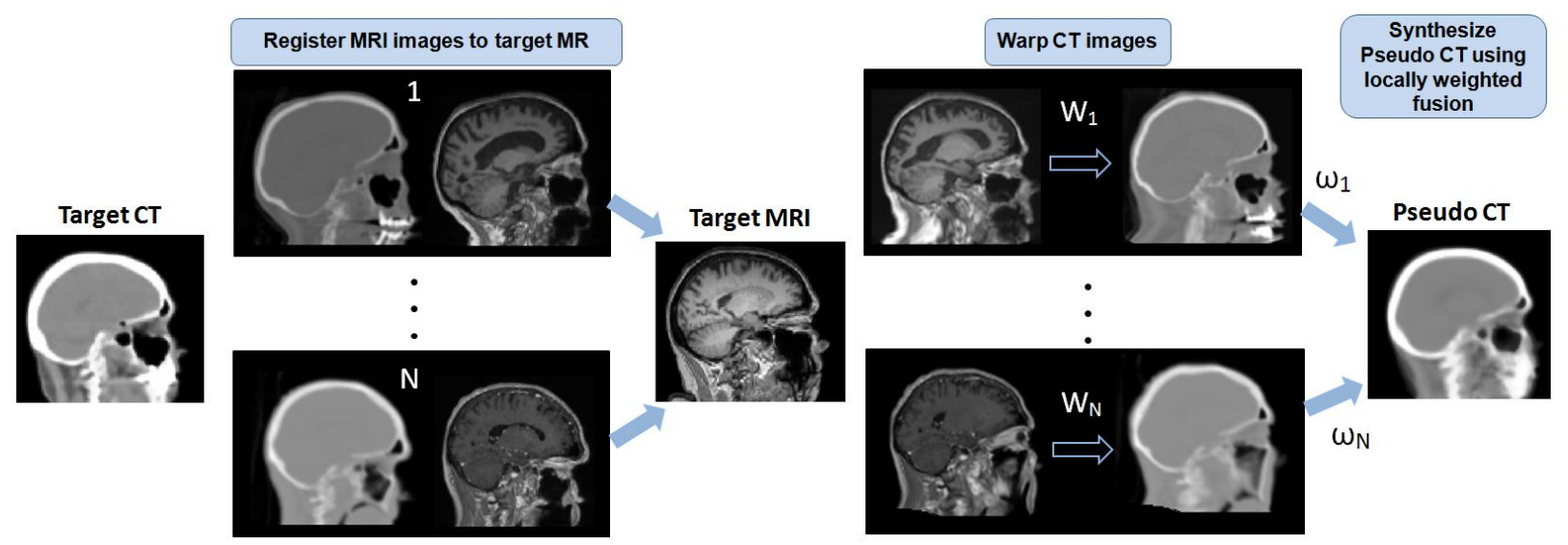

Figure 1. Flowchart of the multi-atlas registration AC method employed in this work. First, all MR images of the atlas dataset are non-rigidly registered one-by-one to the target MR image. Then, their corresponding CT images are wrapped with the same transformation to the target MRI space. Finally, the pseudo-CT is synthesized by local weighting of the wrapped CT images.

\subsubsection{Emission-based AC}

In this work, we employed our recently developed MLAA algorithm to estimate the attenuation map from TOF PET data of the mCT scanner (Mehranian and Zaidi, 2015a). Figure 2 shows the flowchart of the proposed MLAA-GMM algorithm where the activity and attenuation maps are alternatively reconstructed from emission data using a TOF ordinary Poisson ordered subset expectation maximization (OSEM) algorithm and a non-TOF ordered subset maximum likelihood for transmission tomography (OS-MLTR) algorithm. In the OS-MLTR step, two types of priors are imposed on the estimation of attenuation in order to regularize the solution space and therefore address some of the challenges associated with the original MLAA algorithm. To reduce noise in the attenuation estimates, a Markov random field smoothness (MRF) prior with a quadratic potential function is employed. This prior favours attenuation maps that are smooth based on the weighted local differences between voxels in a neighbourhood. To suppress cross-talk artefacts resulting from the propagation of activity features into attenuation maps and vice versa, and more importantly the scaling issue of the MLAA algorithm, a constrained Gaussian mixture model is used. The GMM prior favours an attenuation map whose statistical distribution follows a distribution parameterized over a patient population. This model is based on the observation that the distribution (histogram) of linear attenuation coefficients of 511-keV attenuation maps can be modelled as a mixture of known Gaussian functions with means, standard deviations and mixture proportions calculated from a large number of typical CT datasets. The involved parameters (the mean, standard deviation and mixture propotion of the Gaussian mixture model and the regularization parameters of the GMM and MRF priors) were optimized as described in our previous work (Mehranian and Zaidi, 2015a). 


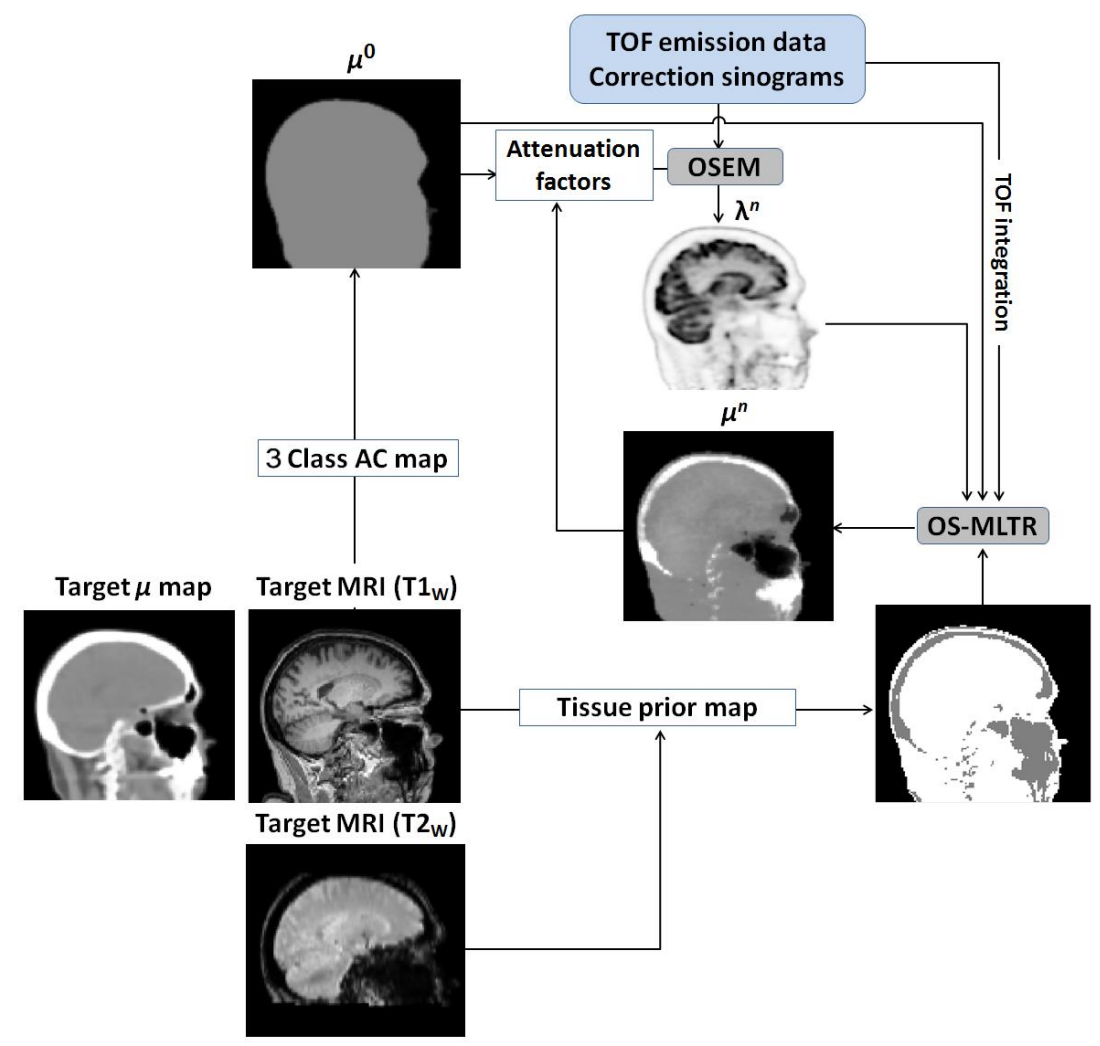

Figure 2. Flowchart of the MLAA-AC algorithm used in this work. This algorithm alternates between an OSEM activity reconstruction and a constrained OS-MLTR transmission reconstruction. The attenuation estimation is guided by an MRI-derived tissue prior map. The algorithm is initialized by a 3-class MRAC attenuation map which is iteratively updated toward the final solution.

Since MRI data provide additional tissue type information, the GMM was spatially unmixed using a tissue prior map obtained from T1- and T2-weighted MRI images. This map indicates the membership of the attenuation voxels to three known tissue classes, namely, soft-tissue, fat and background air, and an unknown tissue class that contains bones, internal air and susceptibility artefacts. For voxels belonging to the known tissue classes, single Gaussians with the known means and standard deviations corresponding to each tissue are employed, while for voxels in the unknown tissue class a mixture of Gaussians is used. The constrained prior penalizes large deviations of attenuation estimation from their expected values (mean LACs) in each tissue class, especially softtissue and therefore substantially suppresses noise, cross-talk artefacts and the unknown scaling of the attenuation maps. Fat and background air tissue classes of the tissue prior map were obtained in a similar way as the 3-class attenuation map described in section 2.2.1. The soft-tissue class was defined as the complement of fat, background air and unknown tissue classes. The unknown class corresponds to the regions that have low MR intensity due to short $\mathrm{T} 2$ relaxation time and low proton density such as bones and air cavities. We employed T2-weighted images as they complete the signal intensity of T1-weighted images over tissues showing low MR intensity due to low T1 relaxation time, such as the cerebrospinal fluid, ventricles and eyes. This enables to correctly include them into the soft-tissue class. The unknown tissue class was therefore obtained by segmentation of superimposed T1- and T2- 
weighted images (which in fact resemble proton-density weighted MR images) using a heuristicallyadjusted thresholding technique.

The proposed MLAA algorithm was implemented for image reconstruction in the native geometry of the mCT scanner. The PET component of the scanner consists of a total number of 32448 LSO crystals, each $4 \times 4 \times 20 \mathrm{~mm}^{3}$ in dimension, accommodated in 4 rings of 48 detector blocks. Time-offlight PET data were acquired using a coincidence window width of $4.06 \mathrm{~ns}$ with an effective TOF timing resolution of $580 \mathrm{ps}$ and sorted into 13 TOF bins with a maximum ring difference of 49 planes. The algorithm was initialized using a uniform activity map and the 3-class attenuation map obtained from tissue classification of T1-weighted images. Scatter sinograms were obtained from TOF singlescatter simulation with the 3-class attenuation maps using the Siemens e7 tools. The activity and attenuation maps were reconstructed using 40 global iterations and 2 and 3 sub-iterations for activity and attenuation, respectively. The regularization parameters of the applied priors were experimentally optimized for 2 clinical studies and used for the rest of patients.

\subsection{Image Reconstruction and Data Analysis}

For each patient, 4 PET image reconstructions were performed using different attenuation maps, including reference CTAC, 3class-AC, MLAA-AC and atlas-AC. The image were reconstructed using Siemens VG50 e7 tool with an OP-OSEM algorithm with point spread function (PSF) modelling and TOF information with 3 iterations and 21 subsets. All data corrections (scatter, random, dead-time, decay, attenuation, and normalization) were included in the reconstruction procedure. The image matrix size is $400 \times 400 \times 109$ with $2 \times 2 \times 2 \mathrm{~mm}^{3}$ voxels.

The accuracy of bones delineation using MLAA-AC and atlas-AC methods was assessed based on bone extraction criteria including the Dice similarity coefficient (DSC), relative volume difference (RVD) and Jaccard similarity (JC) with respect to reference CTAC maps. For each patient, the abovementioned metrics were calculated as follows:

$$
\begin{gathered}
\operatorname{DSC}(A, M)=\frac{2|A \cap M|}{|A|+|M|} \\
\operatorname{RVD}(A, M)=100 \times \frac{|A|-|M|}{|M|} \\
J C(A, M)=\frac{|A \cap M|}{|A \cup M|}
\end{gathered}
$$

where $A$ and $M$ are reference CT-derived and predicted bones, respectively.

The Hermes BRASS analysis tool (Hermes medical solutions $A B$, Sweden) was used for quantitative evaluation of brain PET data. The BRASS software wraps input PET images to the spatial coordinates of an ${ }^{18}$ F-FDG PET template obtained by averaging PET images of 12 normal subjects. The tracer activity quantification is then performed over 63 distinct brain regions located in the left and right hemispheres. In this work, we merged left and right regions, thus reducing the total number of regions to 32. Supplemental figure 1 shows the region map used in our quantitative analysis. 
Quantification errors or bias with respect to reference CTAC PET images were calculated for each region and individual voxels according to the following equation:

$$
B_{i}=\frac{\left(S U V_{m}\right)_{i}-\left(S U V_{C T A C}\right)_{i}}{\left(S U V_{C T A C}\right)_{i}}
$$

where SUV stands for standardized uptake value of the tracer, $i$ indicates region's label number or voxel number and $m$ the method of attenuation correction. Since the PET images were registered to BRASS template's space, we further calculated the mean $(m)$ and standard deviation $(\sigma)$ of the bias across all 14 patients resulting in atlas bias maps according to the following equations (Ouyang et al., 2013):

$$
m_{i}=\frac{\sum_{P=1}^{N} B_{i}^{p}}{N}, \quad \sigma_{i}=\sqrt{\frac{\sum_{P=1}^{N}\left(B_{i}^{p}-m_{i}\right)^{2}}{N-1}}
$$

where $B_{i}^{p}$ is the bias in voxel $i$ for patient $p$ according to Eq. (11). Note that the BRASS software employs a normalized mutual information criterion for robust registration of patient PET images to the PET template. The accuracy of the registration was qualitatively controlled based on the generated temple isocontour to minimize errors induced by miss-alignments.

The correlation between CTAC-PET uptake values and those produced by the studied attenuation correction methods were determined on scatter plots for each of the 32 regions using Pearson correlation analysis. Bland-Altman plots were also used to calculate the concordance between the SUVs and determine the systematic bias introduced by the AC methods. The statistical differences in quantitative performance of the $\mathrm{AC}$ methods were calculated using the paired-sample t-test. The differences were considered statistically significant for a $p$-value less than 0.05 .

\section{RESULTS}

Figure 3 shows the reference CTAC and T1-weighted MR images of two representative patients and compares patient-specific attenuation maps obtained by conventional segmentation-based, emissionbased and atlas registration-based AC methods. For these patients, the tissue prior maps calculated to implement the MLAA-AC method are also shown. The unknown tissue classes, corresponding to MRI voxels with low intensity, are displayed in white colour, while known soft and fat tissue classes are displayed in gray. As shown, the MLAA-AC algorithm can reliably differentiate air cavities from bone/soft-tissues residing in unknown tissue classes. However, this algorithm fails to completely and homogenously estimate bony structures, especially in the temporoparietal regions of the skull. The visual inspection of atlas-AC maps reveals that the atlas-registration method predicts bones more accurately over all regions of the skull compared to the MLAA-AC method. However, it cannot predict well the air cavity's shape, especially in the top-panel patient dataset. The accuracy of bone extraction using these algorithms was objectively evaluated based on metrics defined in Eqs. (8-10). Table 1 summarizes the mean and standard deviation of DCS, RVD and JC metrics calculated for all 
14 clinical studies. Consistent with the qualitative results (figure 3), the quantitative analysis demonstrates that the atlas-AC method outperforms the MLAA-AC method in terms of deriving bones, particularly reflected by an increase of the mean DSC value from 0.79 to 0.92 . The atlas-AC algorithm also improves both RVD and JC metrics as indicators to detect failed segmentation. Figure 4 compares the mean of bone attenuation values of the various attenuation maps per patient. It can be observed that the mean LAC values predicted by the atlas-AC method are generally very close to CTAC reference values, while those predicted by the MLAA-AC method are substantially underestimated. The results show that the means and standard deviations of bone LACs in CTAC, atlas-AC, MLAA-AC and 3-class MRAC maps are, on average, $0.138 \pm 0.012,0.134 \pm 0.02,0.107 \pm$ $0.014,0.097 \pm 0.005 \mathrm{~cm}^{-1}$, respectively. The mean $\pm \mathrm{SD}$ of bone LACs for individual patients are provided in supplemental Table 1. Note that the attenuation maps were smoothed using an isotropic 4 mm Gaussian filter, therefore the bone LACs of 3-class AC method show some variations.

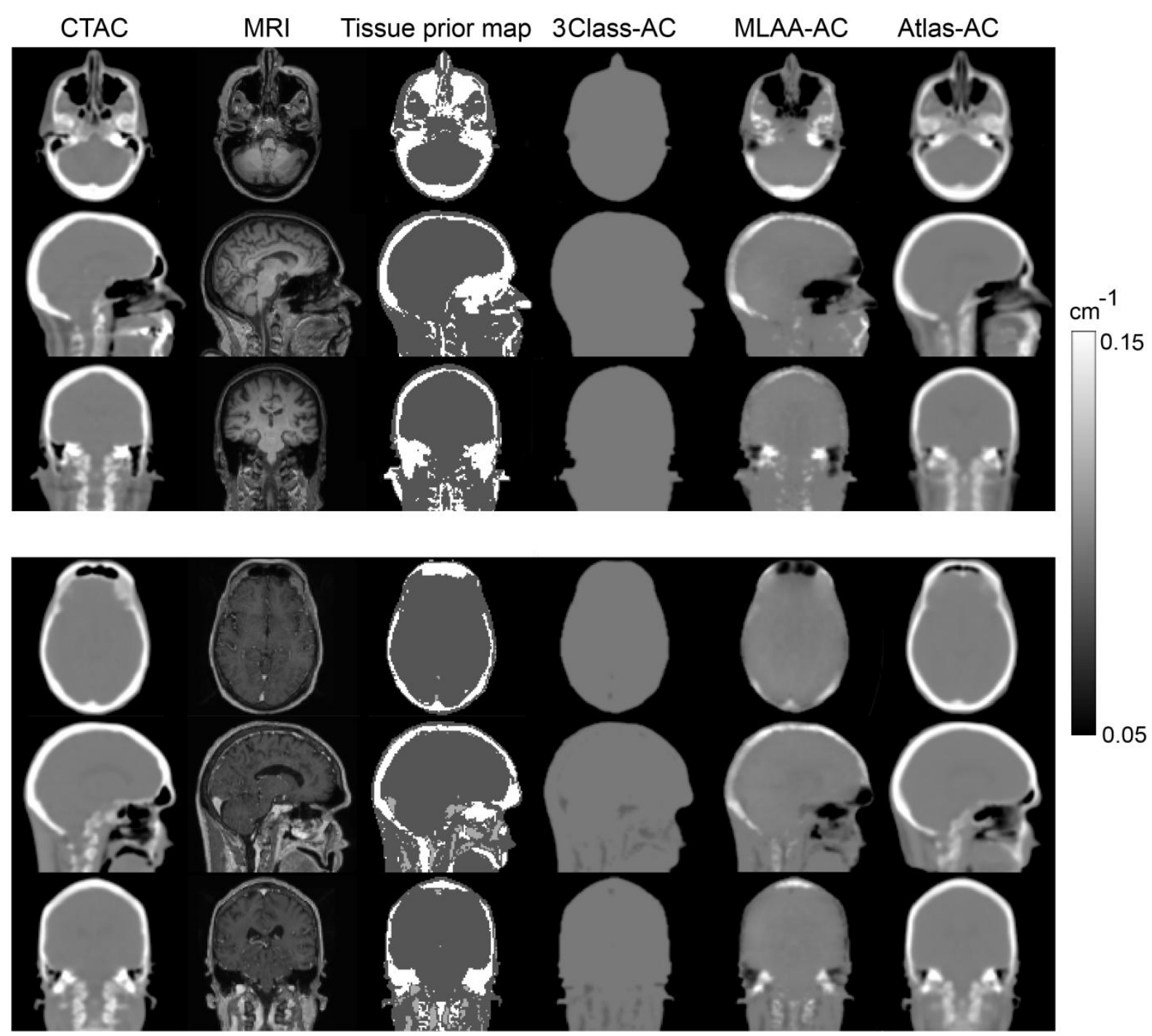

Figure 3. Comparison of the attenuation maps for two representative patients generated by the different attenuation correction techniques together with the reference CTAC map, the target MRI image and MR-based tissue prior map used for the MLAA algorithm. 
Table 1. Quantitative accuracy of the estimated bone tissues by the MLAA-AC and atlas-AC methods over 14 patients (mean \pm standard deviation).

\begin{tabular}{cccc}
\hline Methods & DSC & RVD(\%) & JC \\
\hline MLAA-AC & $0.79 \pm 0.02$ & $-15.6 \pm 2.8$ & $0.64 \pm 0.03$ \\
Atlas-AC & $0.92 \pm 0.02$ & $-9.0 \pm 2.1$ & $0.85 \pm 0.03$ \\
\hline
\end{tabular}

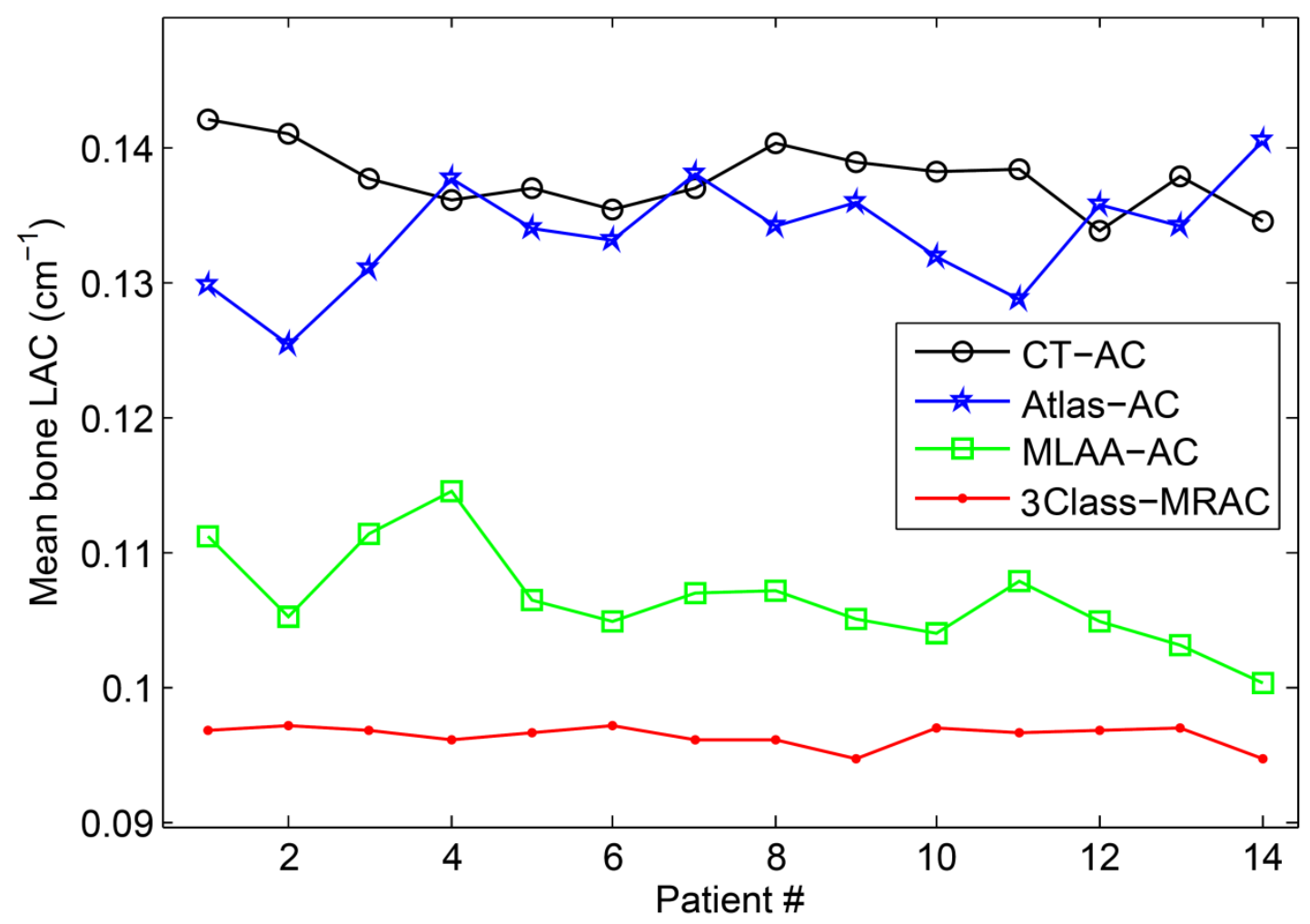

Figure 4. Mean bone linear attenuation coefficients calculated for each patient for different attenuation correction methods.

The quantitative performance of the different $\mathrm{AC}$ methods was further evaluated in comparison with the CTAC method using region-based analysis of PET images warped to fit with the BRASS template space. Supplemental Table 2 summarizes the mean and SD of quantification errors (bias) induced by the 3-class AC, MLAA-AC and atlas-AC methods in 32 regions of the brain over all patients. Figure 5 illustrates the mean of the bias for each region and AC method. The results clearly demonstrate that the atlas-AC method results in a mean positive error $<2 \%$, while the 3 -class $\mathrm{AC}$ and MLAA-AC methods both show high negative errors and thus tracer underestimation over all regions in the brain. It was found that the 3-class AC, MLAA-AC and atlas-AC methods result, on average, in a total bias of $-16.2 \pm 3.6 \%,-13.3 \pm 3.3 \%$ and $1.0 \pm 3.4 \%$, respectively. This magnitude of the bias could be predicted from the mean of the bone LACs shown in figure 4, where both 3-class- and MLAA-AC methods underestimate the bone attenuation coefficients, which is of prime importance in brain PET quantification. As reported in (Andersen et al., 2014), the activity concentration in brain 
regions close to the skull such as occipital gyri and cerebellum is more underestimated by the 3-class AC method.

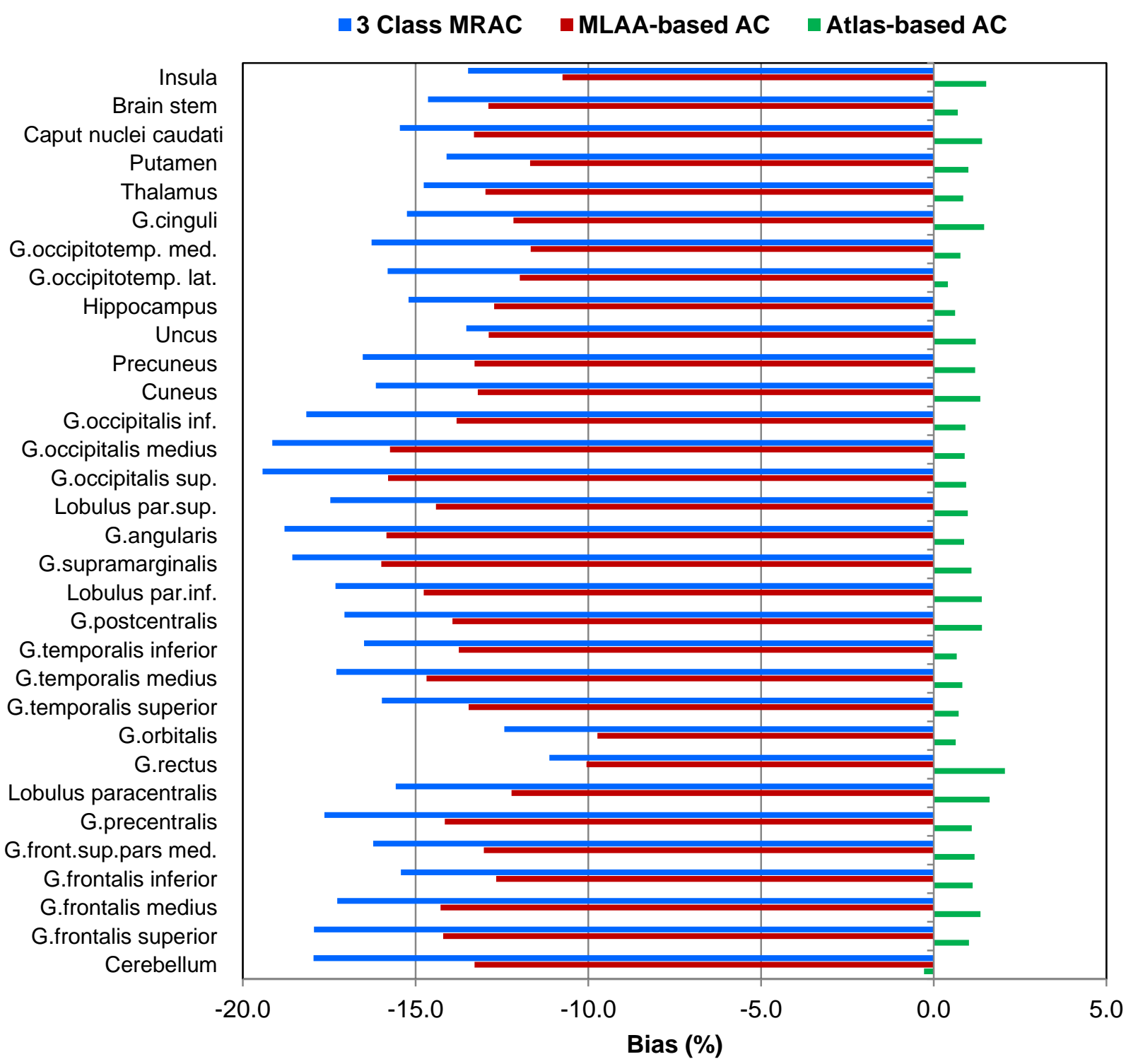

Figure 5. Mean of the PET quantification errors in 32 brain regions calculated over all patients for the different AC methods with respect to reference CTAC.

Figure 6 shows the voxel-wise mean and standard deviation of an atlas bias map calculated for all patients according to Eq. (12). It represents the magnitude and spatial distribution of quantification errors among the studied datasets in axial and sagittal views. Note that due to averaging, mean bias maps do not merely reflect the bias induced by registration errors. The results further support the outperformance of the atlas-AC method by reducing the mean errors especially in or close to bones. However, as also shown in figure 5, this AC method resulted in a slight over-estimation of the tracer concentration in all regions. It can be seen that the MLAA-AC method has slightly improved performance compared to the 3-class AC method. The statistical analysis showed that there is 
significant difference between the performance of the MLAA-AC and 3-class AC methods over most brain regions, except a few regions far from bones such as the precuneus and gyrus rectus $(\mathrm{p}<0.001)$. Similar evaluation between the performance of atlas-AC and the other two methods showed a statistically significant difference. Figure 7 shows linear regression plots illustrating the correlation between the tracer uptake of the different AC methods and the reference CTAC method. The results show that the atlas-AC PET uptake values are highly correlated to CTAC PET values over all 32 brain regions $\left(R^{2}=0.99\right)$. The scatter plots of the 3 -class AC and MLAA-AC PET images show reduced $R^{2}$ correlation coefficients of 0.98 and 0.985 and tend to underestimate the uptake values since the slope of the regression line is less than 1 .

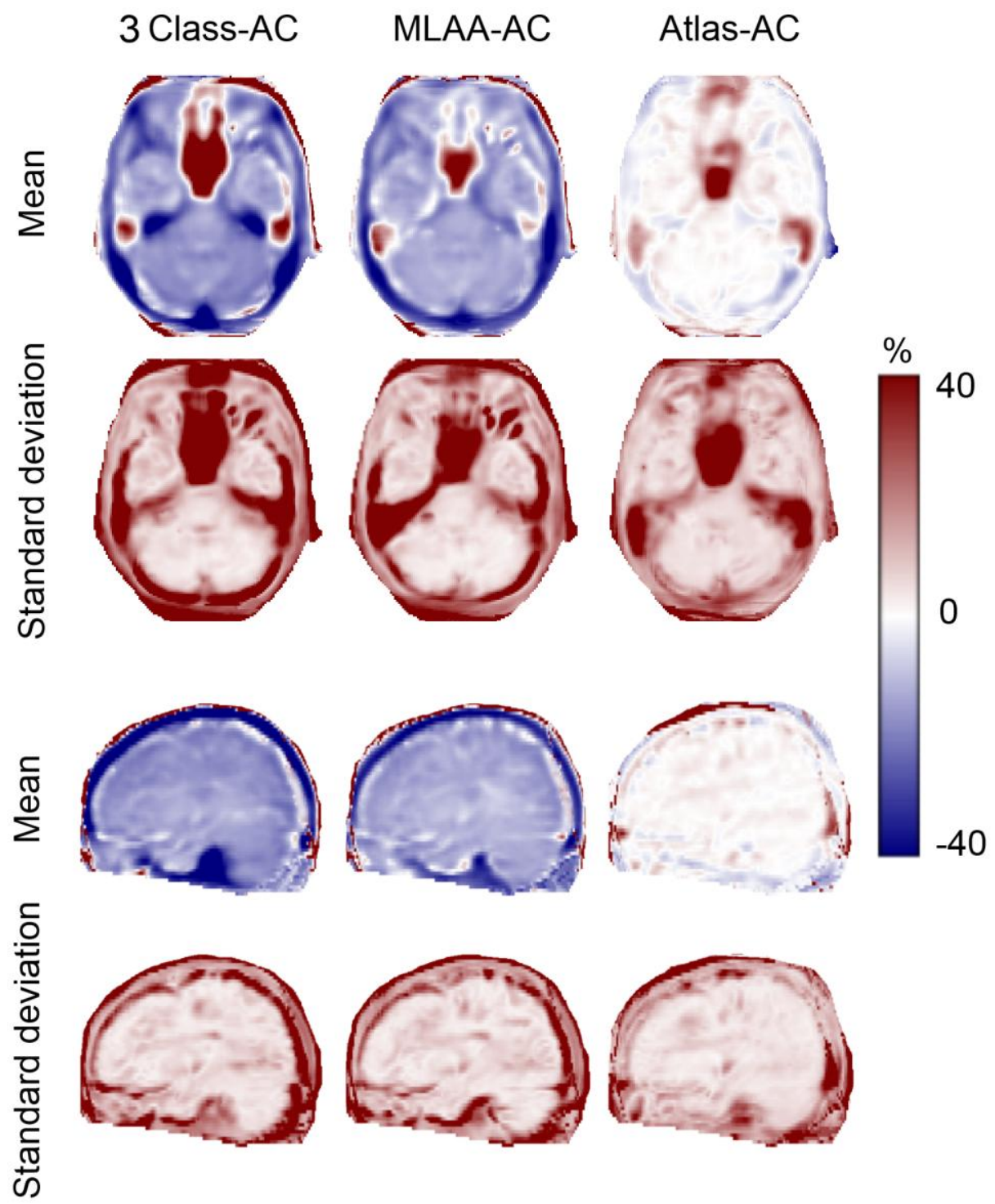

Figure 6. Mean and standard deviation of atlas bias maps calculated over all patients for the different attenuation correction methods displayed in transaxial and coronnal planes. 


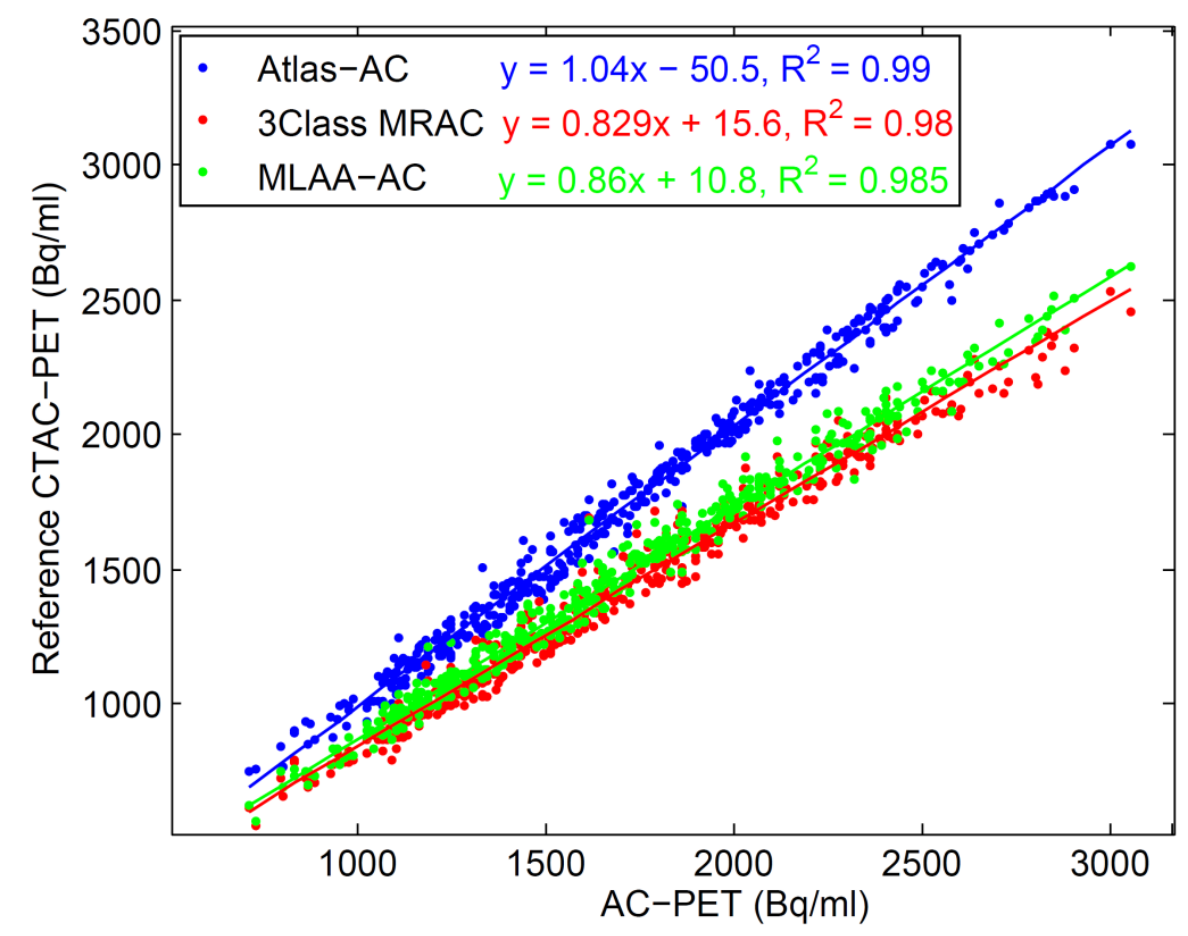

Figure 7. Scatter and linear regression plots between the tracer uptake in PET images reconstructed using reference CTAC maps and those reconstructed using the different AC maps.

Figure 8 shows the results of Bland-Altman concordance analysis. As can be seen, both 3-class and MLAA-AC methods give rise to a high systematic bias in tracer uptake quantification compared to the atlas-AC method. The mean-difference horizontal lines indicating the estimated bias, however, show that the atlas-AC method results in a slight overestimation, as also reflected in pervious results.

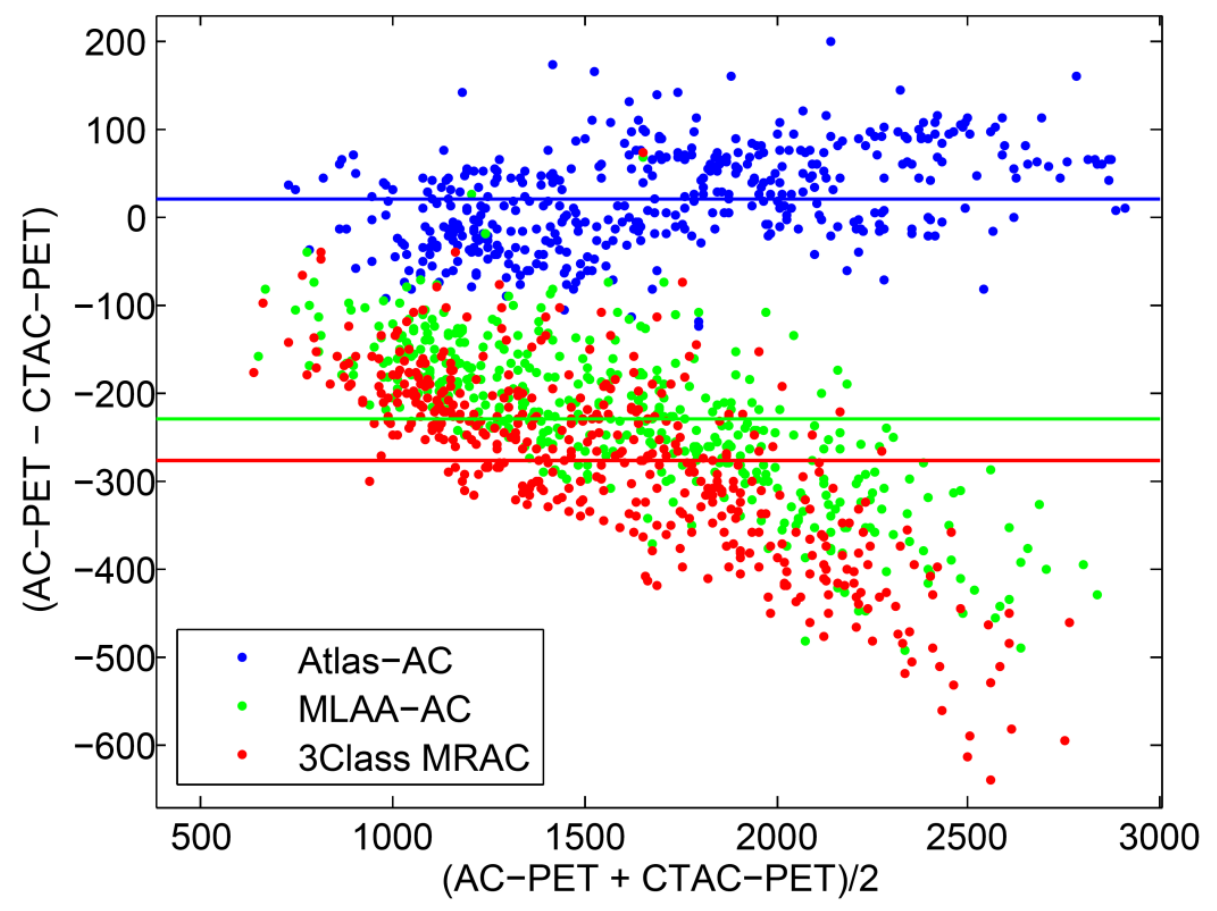

Figure 8. Bland-Altman concordance plots for PET data corrected for attenuation using reference CTAC, 3-class MRAC, MLAA-AC and atlas-AC methods. 


\section{DISCUSSION}

Accurate MRI-guided attenuation correction in PET/MRI has been a major challenge since the introduction of this clinical hybrid imaging technology (Disselhorst et al., 2014). Since then, a number of strategies have been proposed to derive patient-specific attenuation maps either from MR images and PET emission data or co-registered MR/CT datasets. The development of novel AC methods enabling particularly to account for bone attenuation is of importance in PET brain imaging since a large proportion of cortical bone is accommodated in the skull (Zaidi et al., 2007). To provide a clear picture of the performance and cost-effectiveness of these AC methods, we compared the quantitative performance of the standard segmentation-based and state-of-the-art emission- and atlas registrationbased AC methods in brain PET/MR imaging.

The results demonstrate that the exclusion of the bones in 3-class AC methods results in a significant underestimation of tracer uptake, ranging from $13 \%$ to $19 \%$ in various regions of the brain. Consistent with our findings, Dickson et al. also reported underestimations in range of $10-21 \%$ using 25 clinical studies based on a similar regions-wise analysis using SPM templates (Dickson et al., 2014). More importantly, our results showed that in the cortical regions of the brain, such as the cerebellum and frontal gyri, the 3-class AC method results in about $-19 \%$ bias (Table 1), while Dickson et al. reported-21\% bias on average for these regions. These results among others reported in the literature (Hofmann et al., 2008, Izquierdo-Garcia et al., 2014), clearly justify the need for novel more accurate $\mathrm{AC}$ methods that account for bones.

Atlas registration-based methods have therefore been proposed as a viable attenuation correction technique for the prediction of bones from a patient population. In this work, we evaluated the performance of a new multi-atlas registration AC method, which exploits a local weighting atlas fusion strategy. The results of this study showed that this algorithm reduces the bias to $1.0 \pm 3.4 \%$, on average, which is considered as an insignificant error from a clinical perspective. Izquierdo-Garcia $e t$ $a l$. also reported that their atlas-registration method resulted in an error of $0.75 \pm 1.6 \%$ using 16 subjects administered with ${ }^{18} \mathrm{~F}$-FDG and ${ }^{18} \mathrm{~F}$-FET radiotracers (Izquierdo-Garcia et al., 2014). Likewise, Burgos et al. evaluated their improved atlas-AC method for attenuation correction of 22 patients who have undergone ${ }^{18} \mathrm{~F}$-FDG and ${ }^{18} \mathrm{~F}$-florbetapir scans (Burgos et al., 2015). The authors reported that their method resulted in relative mean errors of $<0.6 \%$ for FDG and $<0.1 \%$ for Florbetapir, on average, with a standard deviation of $1 \%$ for both tracers. This study demonstrated that this multi-atlas registration technique outperforms the single-atlas registration AC method proposed by Izquierdo-Garcia et al. (2014).

In spite of promising results, atlas-AC methods have some limitations as their performance depends strongly on the accuracy of the applied registration algorithm, especially in whole-body imaging. In brain PET/MRI, the registration errors are inherently lower than in whole-body imaging owing to the 
high morphological similarity of the brain among different subjects. In addition, the predictive performance of these algorithms depends considerably on how accurately the atlas dataset used for registration and training can represent the morphological variability and pathological abnormalities of the target subject. In other words, the representativeness of the atlas dataset is another key to the success and performance of this category of AC techniques. Due to the limited number of MRI/CT pair datasets in most studies proposing and evaluating atlas-registration based methods, a leave-one out cross-validation approach is used to generate a template or to train the predictive model. However, a limitation of these studies, including the present work, is that the performance assessment of the atlas-AC method on the same datasets used during LOOCV might obscure the actual predictive accuracy of these methods when using different datasets. Therefore, there is a high demand for largescale evaluation of atlas-AC methods in the clinic. The initial promising results of this category of methods in brain PET/MRI has encouraged their adoption on state-of-the-art commercial systems, such as the GE SIGNA TOF PET/MRI scanner, providing an opportunity to validate their robustness and predictive accuracy over a large number of datasets. Recently, Yang et al. compared the performance of the standard atlas-AC method implemented on the commercial scanner with the conventional segmentation-based AC method using 20 clinical studies (Yang et al., 2015). Their initial results demonstrated that the atlas-AC method reduces the average quantification error of the segmentation-based AC method in 8 regions of the brain to less than $5 \%$ (from $9.27 \pm 2.91 \%$ to $4.11 \pm$ $1.68 \%)$.

In this work, we evaluated the performance of the nascent emission-based $\mathrm{AC}$ methods in TOF PET/MRI brain imaging. We employed our previously proposed MLAA-AC algorithm which exploits MRI anatomical and CT statistical information to improve the performance of the original MLAA technique particularly in terms of quantification bias and cross-talk artefacts. Overall, the performance of the MLAA algorithm depends highly on the TOF timing resolution and biodistribution of the PET tracer. The TOF resolution is of particular importance in brain PET imaging. With the current TOF timing resolution (e.g. 580 ps on the Siemens mCT scanner used in this study), most of the detected counts are distributed in a small number of TOF bins. As the TOF resolution improves (e.g. <400 ps for the GE SIGNA PET/MR scanner), the data are further separated in a larger number of TOF bins and therefore the ability of the MLAA algorithms to derive the attenuation experienced by emission data is improved. The results of this study indicate that emission-based methods present a high potential in terms of differentiating air from bone and metallic implants from soft-tissues. However, as shown in Figures 4 and 5, the MLAA-AC algorithm failed to completely estimate bone attenuation and thus to substantially improve the 3-class AC method. However, this category of approaches is in their infancy and still requires more research and development efforts. More recently, an MRconstrained MLAA algorithm was implemented on the GE Signa PET/MR scanner (Ahn et al., 2015). Consistent with our results, the authors demonstrated that the attenuation of metal implants, internal air cavities and bones can be properly estimated using the MLAA algorithm. 
In our initial evaluation of the proposed MLAA-AC algorithm with non-TOF brain PET data (not shown here), we noticed that this algorithm is not only incapable of increasing the value of voxels whose underlying LACs is bone but also reduces their initial soft-tissue values toward air or lung LACs. This can mainly be ascribed to the instability of the non-TOF version of the algorithm despite the precisely-defined MRI/CT constraints. Given that with the current nominal TOF timing resolution of 580 ps the TOF-MLAA algorithm showed promising trends, we expect a substantial improvement of this algorithm on future generation TOF PET/MRI scanners with improved TOF resolution. The performance of the applied MLAA algorithm in deriving bone is somewhat close to what is achieved by UTE-based algorithms since the accuracy of bone segmentation from UTE images is limited by the spatial resolution MR images, which in turn is conditioned by the data acquisition time. Some studies have shown that UTE-segmented bones are usually incomplete and underestimated. Therefore, PET quantification errors are not considerably reduced compared to conventional 3-class MRAC methods (Burgos et al., 2014, Dickson et al., 2014). For instance, Dickson et al. showed that UTE-based AC reduced the quantification errors in the brain from $17 \%$ (for the 3-class MRAC method) to $12 \%$, on average. Our results follow the same trend since the errors of the 3-class AC method were reduced by the MLAA-AC method from about $16 \%$ to $13 \%$. As mentioned above, the performance of emissionbased AC methods also depends on the biodistribution and support provided by the PET tracer. In this work, we included ${ }^{18}$ F-FDG PET studies which is the most widely used tracer for brain imaging in nuclear medicine departments. To demonstrate the influence of the tracer distribution, we analysed one ${ }^{18}$ F-fluoro-ethyl-tyrosine $\left({ }^{18} \mathrm{~F}\right.$-FET) scan. This tracer does not have significant uptake in normal brain tissue but presents with an uptake in the skin and neoplasms such as gliomas. Figure 9 compares the results of the MLAA-AC algorithm for an ${ }^{18}$ F-FDG scan with those of a ${ }^{18}$ F-FET scan. As can be seen, bone attenuation coefficients are more accurately estimated in the ${ }^{18} \mathrm{~F}$-FET study compared to the ${ }^{18} \mathrm{~F}$ FDG scan. These results are in agreement with our previous observations in brain imaging using ${ }^{18} \mathrm{~F}$ FCH (Mehranian and Zaidi, 2015b). Therefore, these observations call for further evaluation of emission-based AC methods with non-FDG tracers. 


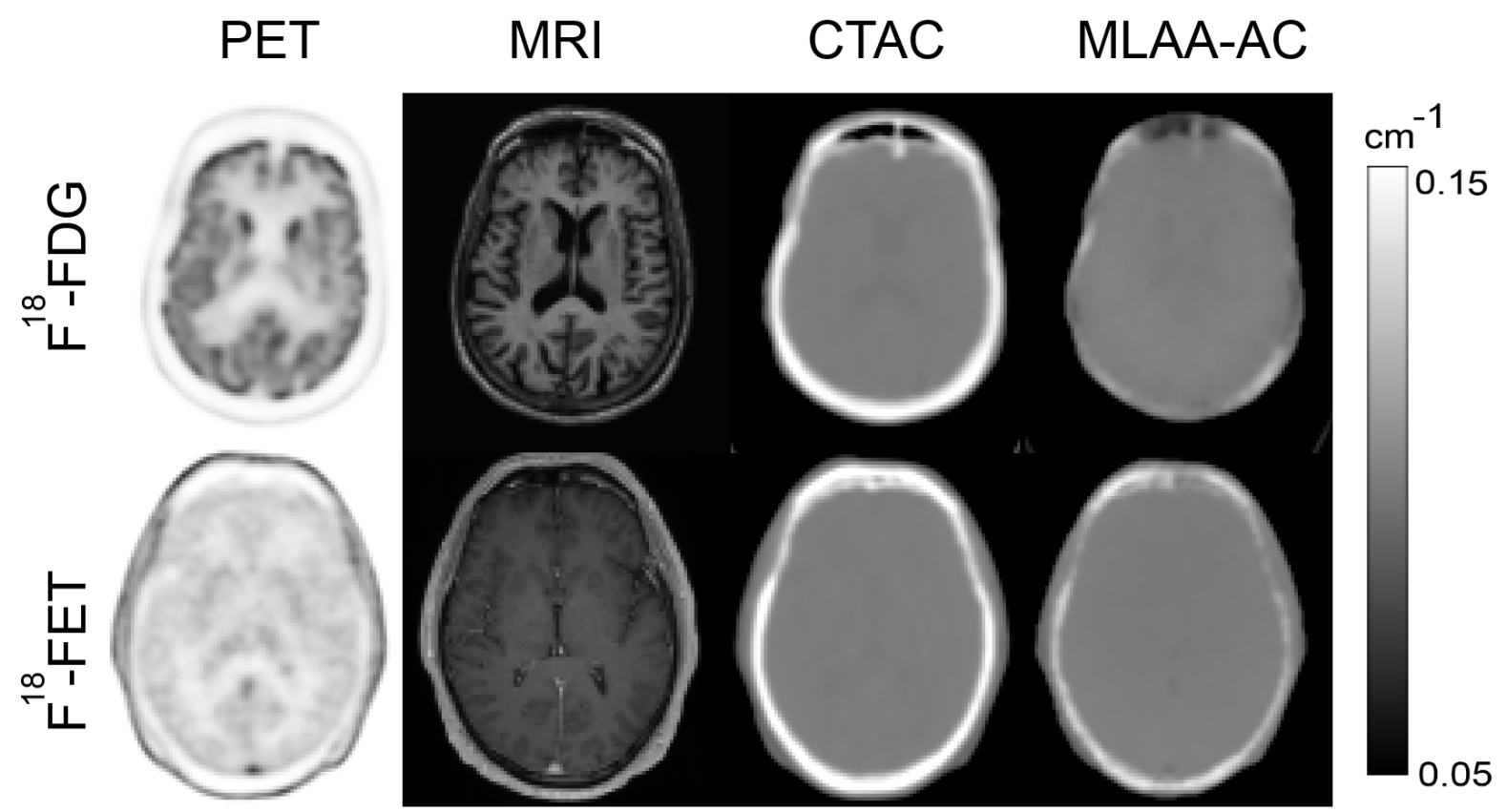

Figure 9. The impact of tracer distribution on the performance of the MLAA-AC algorithm. Note that this algorithm estimate more accurately the bone LACs are from the ${ }^{18}$ F-FET emission data.

One of the limitations of this study is the quantitative analysis of different attenuation correction techniques based on only the relative change of activity concentration (SUV) with respect to reference CTAC-PET data. In neurological studies, it is common practice to normalize PET images with respect to a reference region in the brain, such as the pons (located in brainstem) (Minoshima et al., 1995). Therefore, in addition to SUV analysis, one can also evaluate the AC methods based on the resulting regional SUV (SUVr). In this work, we focused on SUV and absolute tracer quantification metrics, which are widely used in neuro-oncology. Furthermore, the MRI/CT datasets used in this study are chosen out of a large patient population, where patients presenting with highly abnormal anatomy (i.e. due to craniectomy, traumatic brain injury and etc.) or with severe metallic artefacts in dental regions were excluded. Therefore, further investigation is required to scrutinize the performance of atlas- and MLAA-AC algorithms on subjects presenting with pathologies and abnormalities. It should however be noted that in the case of moderate dental metal-susceptibility artefacts, our results showed that both MLAA- and atlas-AC methods are able to reasonably recover high-valued LACs of dental fillings. For instance, the MRI/CT dataset used in Figures 1 and 2 showed moderate metal artefacts in both CT and MR images. As can be seen in these figures, both atlas- and MLAA-AC methods exquisitely estimated the metal's LAC values. The capability of atlas-AC methods should be attributed to the fact that the employed CT/MRI atlas dataset is representative of patients presenting with metal artefacts, since most of the CT images in this dataset were contaminated with metal artefacts. In contrast, the MLAA algorithm recognizes high-valued LACs purely based on the attenuation experienced by emission data along lines of response passing through the metallic objects. Another limitation of this study lies in the biased average age of the included patients toward the elderly (on average 65 years). Most of the 
patients referred to our institution suffer from dementia, which occurs frequently at advanced ages. Therefore, it is also necessary to evaluate the $\mathrm{AC}$ algorithms for different age groups. In particular, the development of age-dependent MRI/CT atlas dataset is of paramount importance for atlas-based pseudo-CT generation in paediatric patients, whose body features different shape and anatomy compared to adults (Bezrukov et al., 2015).

\section{CONCLUSION}

In the present study, the quantitative performance of three generic attenuation correction methods was evaluated in the context of brain TOF PET/MRI imaging. It was found that the segmentation-based AC method results in a signification underestimation of PET tracer uptake in both cortical and subcortical regions of the brain. Our recently developed MRI-guided emission-based AC method, which attempts to estimate bone attenuation values and differentiate them from air cavities, showed promising but limited success in reducing quantification errors. In contrast, the proposed atlas-AC method is capable of predicting more accurate patient-specific attenuation maps, thereby significantly reducing maximum regional quantification errors to about $2 \%$. This work suggests the need for further development of the emission-based AC method for current TOF timing resolution of PET scanners using, for example, empirical functions that promote the derivation of bones. Further evaluation of atlas-registration $\mathrm{AC}$ methods using a large clinical database, which includes patients from different age groups with different pathological indications is guaranteed.

\section{Acknowledgement}

This work was supported by the Swiss National Science Foundation under Grant SNSF 31003A149957 and the Indo-Swiss Joint Research Programme ISJRP-138866.

\section{Conflict of interest}

The authors declare no conflict of interest. 


\section{REFERENCES}

Aasheim, L. B., Karlberg, A., Goa, P. E., Haberg, A., Sorhaug, S., et al. 2015. PET/MR brain imaging: evaluation of clinical UTE-based attenuation correction. Eur J Nucl Med Mol Imaging 42: 1439-1446.

Ahn, S., Cheng, L., Shanbhag, D. D., Wiesinger, F. and Manjeshwar, R. 2015. "Joint reconstruction of activity and attenuation images using MR-based priors: application to clinical PET/MR," In IEEE Nuclear Science Symposium and Medical Imaging Conference (NSS/MIC), San Diego, CA, USA, pp. in press.

Akbarzadeh, A., Ay, M. R., Ahmadian, A., Riahi Alam, N. and Zaidi, H. 2013a. MRI-guided attenuation correction in whole-body PET/MR: assessment of the effect of bone attenuation. Ann Nucl Med 27: 152-162.

Akbarzadeh, A., Gutierrez, D., Baskin, A., Ay, M. R., Ahmadian, A., et al. 2013b. Evaluation of whole-body MR to CT deformable image registration. J Appl Clin Med Phys 14: 238-253.

Andersen, F. L., Ladefoged, C. N., Beyer, T., Keller, S. H., Hansen, A. E., et al. 2014. Combined PET/MR imaging in neurology: MR-based attenuation correction implies a strong spatial bias when ignoring bone Neuroimage 84: 206-16.

Arabi, H., Rager, O., Alem, A., Varoquaux, A., Becker, M., Zaidi, H. 2015. Clinical assessment of MR-guided 3-class and 4-class attenuation correction in PET/MR. Mol Imaging Biol 17: 264-276.

Bezrukov, I., Mantlik, F., Schmidt, H., Scholkopf, B. and Pichler, B. J. 2013. MR-Based PET attenuation correction for PET/MR imaging Semin Nucl Med 43: 45-59.

Bezrukov, I., Schmidt, H., Gatidis, S., Mantlik, F., Schafer, J. F., et al. 2015. Quantitative evaluation of segmentation- and atlas-based attenuation correction for PET/MR on pediatric patients $\mathrm{J} \mathrm{Nucl} \mathrm{Med}$ 56: $1067-74$.

Burgos, N., Cardoso, M., Thielemans, K., Modat, M., Schott, J., et al. 2014. Attenuation correction synthesis for hybrid PET-MR scanners: Application to brain studies. IEEE Trans Med Imaging 33: 2332-2341.

Burgos, N., Cardoso, M. J., Thielemans, K., Modat, M., Dickson, J., et al. 2015. Multi-contrast attenuation map synthesis for PET/MR scanners: assessment on FDG and Florbetapir PET tracers Eur. J. Nucl. Med. Mol. Imag 42: 1447-58.

Carney, J. P., Townsend, D. W., Rappoport, V. and Bendriem, B. 2006. Method for transforming CT images for attenuation correction in PET/CT imaging. Med Phys 33: 976-983.

Catana, C., Drzezga, A., Heiss, W. D. and Rosen, B. R. 2012. PET/MRI for neurologic applications $J$ Nucl Med 53: 1916-25.

Catana, C., van der Kouwe, A., Benner, T., Michel, C. J., Hamm, M., et al. 2010. Toward implementing an MRI-based PET attenuation-correction method for neurologic studies on the MRPET brain prototype. J Nucl Med 51: 1431-1438.

Defrise, M., Rezaei, A. and Nuyts, J. 2012. Time-of-flight PET data determine the attenuation sinogram up to a constant Phys Med Biol 57: 885-899.

Delso, G., Carl, M., Wiesinger, F., Sacolick, L., Porto, M., et al. 2014. Anatomic evaluation of 3- 
dimensional ultrashort-echo-time bone maps for PET/MR attenuation correction J Nucl Med 55: 780785 .

Delso, G., Wiesinger, F., Sacolick, L. I., Kaushik, S. S., Shanbhag, D. D., et al. 2015. Clinical evaluation of zero-echo-time MR imaging for the segmentation of the skull J Nucl Med 56: 417-422.

Dickson, J. C., O'Meara, C. and Barnes, A. 2014. A comparison of CT- and MR-based attenuation correction in neurological PET Eur. J. Nucl. Med. Mol. Imag 41: 1176-89.

Disselhorst, J. A., Bezrukov, I., Kolb, A., Parl, C. and Pichler, B. J. 2014. Principles of PET/MR imaging $\mathrm{J} \mathrm{Nucl} \mathrm{Med} \mathrm{55:} \mathrm{2S-10S.}$

Garibotto, V., Heinzer, S., Vulliemoz, S., Guignard, R., Wissmeyer, M., et al. 2013. Clinical applications of hybrid PET/MRI in neuroimaging. Clin Nucl Med 38: e13-e18.

Hofmann, M., Steinke, F., Scheel, V., Charpiat, G., Farquhar, J., et al. 2008. MRI-based attenuation correction for PET/MRI: a novel approach combining pattern recognition and atlas registration $J \mathrm{Nucl}$ Med 49: 1875-1883.

Izquierdo-Garcia, D., Hansen, A. E., Forster, S., Benoit, D., Schachoff, S., et al. 2014. An SPM8based approach for attenuation correction combining segmentation and nonrigid template formation: application to simultaneous PET/MR brain imaging J Nucl Med 55: 1825-30.

Jacobs, A. H., Kracht, L. W., Gossmann, A., Ruger, M. A., Thomas, A. V., et al. 2005. Imaging in neurooncology NeuroRx 2: 333-47.

Keereman, V., Fierens, Y., Broux, T., De Deene, Y., Lonneux, M., et al. 2010. MRI-based attenuation correction for PET/MRI using ultrashort echo time sequences $J$ Nucl Med 51: 812-8.

Klein, S., Staring, M., Murphy, K., Viergever, M. A. and Pluim, J. P. 2010. elastix: a toolbox for intensity-based medical image registration IEEE Trans Med Imaging 29: 196-205.

Kops, E. R., Wagenknecht, G., Scheins, J., Tellmann, L. and Herzog, H. (2009) In IEEE Nuclear Science Symposium Conference Record (NSS/MIC), pp. 2530-2533.

Kovesi, P. 2000. Phase congruency: A low-level image invariant Psychological research 64: 136-148.

Martinez-Möller, A., Souvatzoglou, M., Delso, G., Bundschuh, R. A., Chefd'hotel, C., et al. 2009. Tissue classification as a potential approach for attenuation correction in whole-body PET/MRI: evaluation with PET/CT data J Nucl Med 50: 520-526.

Mehranian, A., Arabi, H. and Zaidi, H. 2015. Magnetic resonance imaging-guided attenuation correction in PET/MRI: challenges, solutions and opportunities Med Phys under revision

Mehranian, A. and Zaidi, H. 2015a. Clinical assessment of emission- and segmentation-based MRIguided attenuation correction in whole body TOF PET/MRI. J Nucl Med 56: 877-883.

Mehranian, A. and Zaidi, H. 2015b. Joint estimation of activity and attenuation in whole-body TOF PET/MRI using constrained Gaussian mixture models. IEEE Trans Med Imaging 34: 1808-1821.

Minoshima, S., Frey, K. A., Foster, N. L. and Kuhl, D. E. 1995. Preserved pontine glucose metabolism in Alzheimer disease: a reference region for functional brain image (PET) analysis $J$ Comput Assist Tomogr 19: 541-7. 
Montandon, M.-L. and Zaidi, H. 2005. Atlas-guided non-uniform attenuation correction in cerebral 3D PET imaging. Neuroimage 25: 278-286.

Nuyts, J., Dupont, P., Stroobants, S., Benninck, R., Mortelmans, L., et al. 1999. Simultaneous maximum a posteriori reconstruction of attenuation and activity distributions from emission sinograms IEEE Trans Med Imaging 18: 393-403.

Ortiz, C. G. and Martel, A. 2012. Automatic atlas-based segmentation of the breast in MRI for 3D breast volume computation Medical physics 39: 5835-5848.

Ouyang, J., Se Young, C., Petibon, Y., Bonab, A. A., Alpert, N., et al. 2013. Bias atlases for segmentation-based PET attenuation correction using PET-CT and MR IEEE Trans Nuc Sci 60: 33733382.

Rezaei, A., Defrise, M., Bal, G., Michel, C., Conti, M., et al. 2012. Simultaneous reconstruction of activity and attenuation in Time-of-Flight PET IEEE Trans Med Imaging 31: 2224-33.

Salomon, A., Goedicke, A., Schweizer, B., Aach, T. and Schulz, V. 2011. Simultaneous reconstruction of activity and attenuation for PET/MR IEEE Trans Med Imaging 30: 804-813.

Samarin, A., Burger, C., Wollenweber, S., Crook, D., Burger, I., et al. 2012. PET/MR imaging of bone lesions - implications for PET quantification from imperfect attenuation correction Eur. J. Nucl. Med. Mol. Imaging 39: 1154-1160.

Tustison, N. J., Avants, B. B., Cook, P. A., Yuanjie, Z., Egan, A., et al. 2010. N4ITK: Improved N3 bias correction IEEE Trans Med Imaging 29: 1310-1320.

Wiesinger, F., Sacolick, L. I., Menini, A., Kaushik, S. S., Ahn, S., et al. 2015. Zero TE MR bone imaging in the head. Magn Reson Med in press

Yang, J., Jian, Y., Tohme, M., Behr, S., Vigneron, D., et al. 2015. "Impact of atlas-CT-based bone anatomy compensation on MR-based attenuation correction for brain PET imaging in a time-of-flight PET/MRI system: A direct comparison to a patient-CT-based approach," Proceedings of the 4th PSMR Conference on PET/MR and SPECT/MR. EJNMMI Physics (Suppl 1: A68), Eleba, Italy.

Zaidi, H. 2007. Is MRI-guided attenuation correction a viable option for dual-modality PET/MR imaging? Radiology 244: 639-642.

Zaidi, H., Montandon, M.-L. and Meikle, S. 2007. Strategies for attenuation compensation in neurological PET studies. Neuroimage 34: 518-541. 


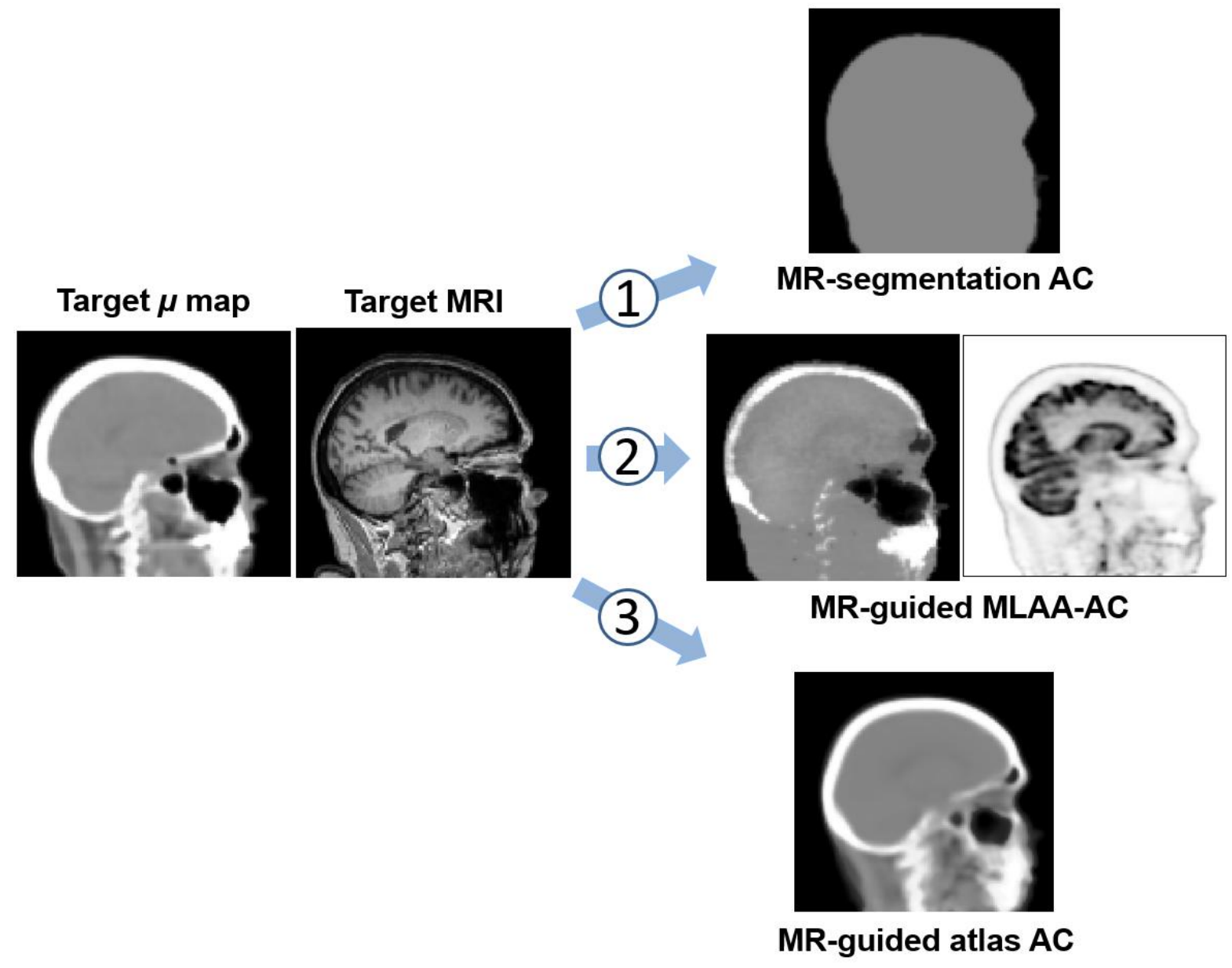

